\title{
Treatment of odontogenic keratocysts by enucleation with adjunctive therapy has lower recurrence rates
}

\author{
What treatment is best for people who have odontogenic keratocysts?
}

\begin{abstract}
Blanas N, Freund B, Schwartz M, Furst I M. Systematic review of the treatment and prognosis of the odontogenic keratocyst. Oral Surg Oral Med Oral Pathol Oral Radiol Endod 2000; 90:553-558

Data sources Index Medicus, MEDLINE were searched, as well as the bibliographies of identified studies.

Study selection Papers selected were those in which odontogenic keratocysts (OKC) was diagnosed histologically, the patient selection process was adequately described, there was consecutive, adequate description of the follow-up period, and where detailed treatment information and recurrence rates were included and assessed using eight defined criteria.
\end{abstract}

Data extraction and synthesis The treatment method and recurrence rates were extracted and tabulated.

Results Fourteen papers met the criteria. These were restricted to retrospective consecutive case-series. The type of treatment and recurrence rates are shown in the Table 1.

Conclusions The literature is limited to retrospective study designs and prospective studies need to be initiated. It appears, however, that resection or enucleation with adjunctive therapy is associated with lower recurrence rates than enucleation alone.

Table 1. Recurrence rates by treatment type.

\begin{tabular}{lcc}
\hline Treatment type & Cysts reported & No. recurrences (\%) \\
\hline Curettage & 26 & $5(19.2)$ \\
Enucleation alone & 387 & $111(28.7)$ \\
Enucleation and Carnoy's* & 60 & $1(1.6)$ \\
Radical enucleation & 6 & $1(16.7)$ \\
Enucleation and cryotherapy & 16 & $5(31.3)$ \\
Marsupialisation & 45 & $11(24.4)$ \\
Resection & 38 & $0(0)$ \\
\hline
\end{tabular}

*Carnoy's solution (glacial acetic acid, ferric chloride, chloroform and absolute alcohol).

Address for correspondence: lan Furst, 697 Coronation Blvd, Cambridge, Ontario, Canada N1R 3GS. E-mail: ian_furst@yahoo.com

\section{Commentary}

OKC is a term coined in the mid-1950s by oral pathologists in Europe to denote a cyst with particular histopathological features and clinical behaviour. It is generally agreed that it arises from the cell rests of the dental lamina, ie, the dental primordium. The term OKC was used synonymously with the term "primordial cyst", which had appeared in earlier literature and classifications, describing a cyst that occurs in place of a tooth, originating from the cystic degeneration of the enamel organ before the development of the dental hard tissues. ${ }^{1}$ The 1971 World Health Organisation (WHO) classification used primordial cyst as the preferred terminology. ${ }^{2}$ The second edition of the WHO classification, which was published in 1992, nevertheless used "odontogenic keratocyst" as its designation ${ }^{3}$ and this has now become the established term.

OKC represents a distinctive developmental odontogenic cyst that requires special consideration because of its unique histopathological features and locally aggressive behaviour. These cysts have marked tendency to recur. ${ }^{3}$ As such, there were suggestions that $\mathrm{OKC}$ be regarded as a benign cystic neoplasm rather than cyst. Furthermore, some people experience multiple odontogenic cysts and such patients should have nevoid basal cell carcinoma (Gorlin) Syndrome (NBCCS) excluded; this is an autosomal dominant inherited condition comprising multiple basal cell carcinomas of skin, OKC, intracranial calcification and bifid rib as well as vertebral anomalies. Prevalence of this syndrome is estimated to be about 1 in $60000 .^{1}$

In view of the marked recurrence rate, treatment of $\mathrm{OKC}$ has always been challenging. As with most cysts, OKC is usually treated by enucleation and curettage. Complete enucleation is difficult, however, because of the thin, friable nature of the cyst wall. This has contributed to the reported frequency of recurrence, ranging from 5 to $62 \%$. This wide variation in recurrence rate is thought to be due to the myriad treatment approaches, ranging from simple curettage to resection of the affected jaw.

This review by Blanas et al. is a very welcome and timely attempt to provide an evidence-base for the various treatment modalities employed to treat OKC. It addresses the fairly focused question of whether there is one management approach that would give the best treatment and prognosis for patients with OKC. This relatively comprehensive, well-executed systematic review provides fairly sound evidence to answer the question posed. A detailed search strategy identified 2290 reports of which 14 met the exclusion and inclusion criteria. The assessment of relevance and validity as well as the data extraction was carried out independently by four individual assessors. The results are valid and interpreted appropriately, but the level of evidence produced by this systemic review is not high (level 4 at most) because all the papers so far published are retrospective, including the 14 selected for this review.

Randomised controlled trials of the management of OKC are lacking and the treatment provided depends very much on the training, experience and management philosophy of the 
institutions in different parts of the world. Although treatment by resection appeared to give the best prognosis, with no recurrences in 38 out of the 578 OKC treated in this way (6.6\% of the total), most surgeons and oral pathologists were ill at ease recommending this to people with OKC. This is because of the benign nature of the disease and the relatively low recurrence rate associated with the other, less invasive, procedures. The invasive nature of resection and reconstruction of the mandible or maxilla and the resultant nerve paraesthesia and increased morbidity cannot necessarily be justified therefore.

The heterogeneity between studies was discussed by the authors and it would not be appropriate to apply a uniform quantitative analysis to such non-uniform data. Despite the shortcomings of reviewing respective case-series, this systematic review does, however, provide evidence that would modify clinical practice. A future prospective study might address the difficulties in a retrospective analysis by better controlling the variables that were identified in this systematic review (ie, variations in treatment, different locations of cysts, presence or absence of infection, size of lesion, associated teeth, mucosa involvement and association with NBCCS). In conclusion, the results are applicable to patients with $\mathrm{OKC}$ and the preferred modality appear to be enucleation with application of Carnoy's solution.

\section{Practice point}

- Although evidence supports enucleation with application of Carnoy's solution as for treatment of OKC, prospective studies are still required.

\section{Jin Fei Yeo}

Department of Oral and Maxillofacial Surgery, National University of Singapore, Singapore

1. Neville BW, Damm DD, Allen CM, Bouquot JE. Oral and Maxillofacial Pathology. 2nd Edn. Philadelphia: WB Saunders Company; 2002.

2. Pindborg JJ, Kramer IRH. International Histological Classification of Tumours. No. 5. Histological Typing of Odontogenic Tumours, Jaw Cysts and Allied Lesions. Geneva: World Health Organisation; 1971.

3. Kramer IRH, Pindborg JJ, Shear M. International Histological Classification of Tumours. No. 5. Histological Typing of Odontogenic Tumours, Jaw Cysts and Allied Lesions. 2nd Edn. Geneva: World Health Organisation; 1992.

Evidence-Based Dentistry (2003) 4, 53-54.

doi:10.1038/sj.ebd.6400203 